\title{
Artigos
}

\section{Quale educazione da zero a sei anni? Prospettive legislative e nuove sfide culturali per i servizi educativi italiani}

Riepilogo: L'articolo presenta e analizza la nuova prospettiva posta dal "Sistema integrato di educazione e istruzione dalla nascita ai sei anni" definita dalla Legge 107/2015 e dal Decreto n.65 del 2017. I servizi educativi per la prima infanzia e la scuola dell'infanzia compongono un sistema integrato in cui, a partire da una nuova visione del bambino da zero a sei anni, avrà particolare importanza il tema della continuità educativa e di una cultura pedagogica condivisa tra nido e scuola dell'infanzia. La nuova legge apre importanti prospettive educative nell'ottica dello zero-sei ma solleva anche nuove sfide che riguardano in particolare le modalità di coordinamento e i processi di formazione della professionalità di educatori e insegnanti.

Parole chiave: Politiche per l'infanzia. Servizi educativi.

\section{Qual educação de zero a seis anos? Perspectivas legislativas e novos desafios culturais para a educação infantil italiana}

\begin{abstract}
Resumo: O artigo apresenta e analisa a nova perspectiva posta pelo "Sistema Integrado de Educação e Instrução do nascimento até os seis anos", definida pela Lei 107/2015 e pelo Decreto 65 de 2017. A educação infantil para a primeira infância e a pré-escola compõem um sistema integrado que a partir de uma nova visão da criança de zero a seis anos trará relevância ao tema da continuidade educativa e de uma cultura pedagógica condizente com a creche e a pré-escola. A nova lei abre uma importante perspectiva educativa sob a ótica de zero a seis, mas também aumenta os novos desafios, particularmente no que se refere à coordenação e aos processos de formação profissional dos educadores e professores.
\end{abstract}

Palavras-chave: Políticas para a pequena infância. Educação Infantil

\section{What education from zero to six years? Legislative perspectives and new cultural challenges for Italian early childhood education/ educational services}

Abstract: The paper presents and analyzes the new perspective from the "Integrated Education and Education System from birth to six years" defined by Law 107/2015 and Decree No.65 of 2017. Early Childhood education for toddler 0-3 and 3-6 pre school Childhood are an integrated system in which, starting with a new vision of the child from zero to six years, the theme of educational continuity and a pedagogical culture shared between "nursery" and pre school will be of particular importance. The new law opens up important educational perspectives in the perspective of zero-six, but also raises new challenges, particularly regarding coordination and training processes of professional educators and teachers.

Keywords: Childhood and Childcare policies. Early childhood education. Educational senvices

\footnotetext{
1 Doutora em Pedagogia Generale e Sociale. Professora na Università degli Studi di Milano-Bicocca. E-mail: agnese.infantino@unimib.it
} 


\section{Verso il sistema integrato 0-6}

$\mathrm{I}$

nido e la scuola dell'infanzia in Italia si sono sviluppati secondo linee e traiettorie specifiche, che hanno disegnato realtà con identità ben distinte, con storie, tradizioni educative e confini chiaramente delineati e differenziati. Come si sa il nido, nato con la legge 1044 del 1971 a seguito dell'azione politica animata "dal basso", dal sindacato e dai movimenti femministi per tutelare il diritto delle donne lavoratrici, era ed è tuttora un servizio a domanda individuale, i cui costi sono quasi interamente coperti dalle rette a carico delle famiglie che ne beneficiano, ed è un servizio in capo ai Comuni sia che se ne occupino in forme di gestione diretta sia che optino per forme di gestione indiretta con l'intervento di imprese appartenenti al mondo del privato sociale. Le Regioni, cui compete la definizione dei confini normativi per la regolamentazione del funzionamento del servizio, hanno generato in questi anni un panorama fortemente differenziato e disomogeneo lungo il territorio nazionale sia per quanto riguarda i criteri di funzionamento, con forti differenziazioni da regione e regione, sia per gli scarti significativi nella diffusione del servizio quasi del tutto assente nelle regioni meridionali del Paese. Ciò pone seri problemi alle politiche di sviluppo del sistema educativo chiamate a trovare soluzioni efficaci per garantire il rispetto dei diritti di accesso ai servizi da parte di tutti i bambini indipendentemente dal territorio in cui vivono. Se alla sua nascita il nido ha risposto prevalentemente al diritto delle madri lavoratrici, oggi è invece il tema dei diritti dei bambini a emergere in modo molto chiaro. I servizi educativi si trovano di fronte alla necessità di riconoscere e rispettare il diritto del bambino alla cura, all'educazione e al riconoscimento ad essere considerato persona intera, non sezionata in funzione dell'età, delle fasi di sviluppo, degli stadi dell'apprendimento. (Cagliari, 2017) In un'epoca in cui la politica stenta a elaborare risposte coerenti ai gravi problemi dello sviluppo culturale, economico, sociale, e vacilla sotto le sfide poste dai nuovi volti delle povertà, del divario sociale, dell'uso smisurato delle risorse, non è affatto semplice tenere alta l'attenzione dell'agenda politica sui temi dell'infanzia e della qualità dei contesti familiari, dei servizi, degli ambienti urbani che caratterizzano le esperienze dei primi anni di vita dei bambini. Un tentativo di fornire risposta all'insufficiente offerta di servizi educativi nella fascia zero-tre anni è stato messo in atto con la sperimentazione delle sezioni primavera, avviate nel 2007 principalmente per tentare di far fronte, a costi contenuti, alla diffusione di nuovi servizi educativi prevedendo in particolare la possibilità per i bambini dai ventiquattro mesi di frequentare un servizio educativo attivato all'interno di strutture scolastiche esistenti, quelle della scuola dell'infanzia. Ne sono derivate esperienze molto differenziate - che meritano una riflessione specifica, che esula da questa riflessione - da cui hanno preso forma nuove opportunità di avvicinamento e dialogo tra due segmenti dell'offerta educativa rivolti alla fascia zero-sei anni (il nido e la scuola dell'infanzia) che, tranne in alcune isolate realtà, solitamente non condividono, e non hanno condiviso nemmeno in passato, una comune cultura dell'infanzia. Forse a 
rendere più evidente questa distanza ha influito anche la differente storia di questi due segmenti educativi. La scuola dell'infanzia infatti, pur essendo un servizio non obbligatorio come il nido, ha avuto una buona diffusione su tutto il territorio nazionale forse perché affidata, fin dal momento della sua istituzione, alla competenza statale, ovvero al Ministero della Pubblica Istruzione. Pochi Comuni hanno scelto la strada di gestire scuole dell'infanzia, è significativa in questo senso l'esperienza di importanti comuni del Nord, ad esempio Milano, Mantova, Brescia per considerare il solo territorio lombardo. La scuola dell'infanzia, sia nella sua espressione pubblica che in quella privata (in particolare per iniziativa cattolica) è strutturata nel rispetto delle "Indicazioni nazionali per il curricolo" che, richiamando espressamente i principi costituzionali, assegnano alla persona un ruolo centrale nella cultura e nelle finalità della scuola e riconoscono nei bambini individui portatori di specifici diritti. Infatti, come affermano le Indicazioni, "La scuola dell'infanzia, statale e paritaria, si rivolge a tutte le bambine e i bambini dai tre ai sei anni di età ed è la risposta al loro diritto all'educazione e alla cura, in coerenza con i principi di pluralismo culturale ed istituzionale presenti nella Costituzione della Repubblica, nella Convenzione sui diritti dell'infanzia e dell'adolescenza e nei documenti dell'Unione Europea". (Indicazioni Nazionali 2012) Questo breve panorama aiuta a visualizzare la portata delle novità poste dalla Legge 107 del 2015 e nello specifico dal Decreto Legislativo del 13 aprile 2017 n. 65 relativo all' "istituzione del sistema integrato di educazione e di istruzione dalla nascita sino ai sei anni". Il decreto, proprio con l'istituzione di un sistema integrato di educazione e istruzione da zero a sei anni, apre nuove opportunità e sfide di non poco conto. La prima e rilevante implicazione riguarda il passaggio del nido e dei servizi educativi per l'infanzia nell'ambito delle competenze del Ministero della Pubblica Istruzione, in un dialogo diretto e in una chiara logica di continuità con la scuola dell'infanzia. La finalità del sistema integrato è infatti di "promuovere la continuità del percorso educativo e scolastico, con particolare riferimento al primo ciclo di istruzione, sostenendo lo sviluppo delle bambine e dei bambini in un processo unitario, in cui le diverse articolazioni del Sistema integrato di educazione e istruzione collaborano attraverso attività di progettazione, di coordinamento e di formazione comuni" (Decreto 65, art. 1, 3 a) Si tratta di un passaggio importante e significativo per la storia del nido e dei servizi educativi per la prima infanzia, che incide profondamente nella ridefinizione del mandato e dell'identità culturale e educativa di questi servizi. Se indubbiamente anche la scuola dell'infanzia non può che essere interessata e coinvolta dalla definizione di un sistema integrato zero-sei, va rilevato come, perlomeno in questa prima fase, le novità più profonde interessino prevalentemente il nido e i servizi educativi che transitano nell'ambito di un Ministero che finora non si era mai occupato di questi servizi, e entrano così in contatto diretto, a tutti gli effetti, con il mondo della scuola, della formazione e dell'istruzione. L'altro significativo passaggio riguarda la progressiva definizione di un sistema integrato zero-sei e di una nuova cultura dell'infanzia che non può essere, a mio parere, l'esito di una pura sommatoria della cultura del nido e di quella della scuola dell'infanzia, alla ricerca di improbabili e inutili mediazioni e equilibri, ma rinvierà alla generazione di altri processi culturali, tutti da esplorare e inventare nei prossimi anni. Ma a questo si arriverà con il tempo. Il primo significativo passaggio di svolta in questa fase interroga prevalentemente, e a vario livello, il nido: come si ridefinirà il suo mandato 
istituzionale? Come sarà declinato il tema degli apprendimenti, dei saperi e della costruzione della conoscenza nelle prime età della vita? Tra cura e educazione ci sarà spazio per elaborare anche altri approcci allo sviluppo dei bambini piccoli? Evidentemente non sono questioni di poco conto.

\section{Um nuovo nido?}

E' quindi la cultura del nido ad essere prevalentemente sollecitata da questo nuovo scenario. Nel mondo della ricerca e nella cultura educativa maturata nei servizi educativi per la prima infanzia in questi anni è sempre stato vivo un intenso dibattito tra posizioni teoriche e punti di vista educativi che tematizzano in modo diverso tanto la vita e la crescita dei bambini nei primi anni di vita quanto la progettualità e il significato educativo dei servizi. In una rappresentazione estremamente schematica e riduttiva si potrebbe affermare che all'interno di questo dibattito è possibile individuare due posizioni distinte che, in modo più o meno accentuato e radicalizzato, insistono l'una nel proporre un'immagine di bambino prevalentemente inteso come essere relazionale, implicato fin dalla nascita in dinamiche relazionali improntate alla reciprocità con gli adulti significativi per il suo mondo emotivo, in grado di uno sviluppo positivo a condizione che intorno a lui si sviluppi un sistema di cure coerente, stabile e prevedibile; l'altra incline a evidenziare in particolare il bambino cognitivo, dotato fin dalla nascita di potenzialità socio-cognitive che, per poter avere pieno corso, impegnano gli adulti nella predisposizione intenzionale di contesti di sviluppo e opportunità di apprendimento a misura dei bambini, delle modalità con cui essi stessi, in percorsi originali, sono in grado di produrre conoscenza. Si potrebbe affermare che la "pedagogia della cura" e la "pedagogia degli apprendimenti", soprattutto nelle pratiche educative dei servizi e nel pensiero degli educatori, si siano via via sempre più polarizzate su versanti opposti, anche ben al di là delle intenzioni di chi si occupa teoricamente di questi temi, e, così polarizzate, non siano riuscite a entrare in un fruttuoso dialogo $\mathrm{e}$ in una visione omogenea dello sviluppo tanto che "cura" e "apprendimenti" sono spesso ritenuti due distinti paradigmi e approcci all'educazione del tutto antitetici e dicotomici, reciprocamente escludentisi, non conciliabili. Molti educatori, a partire dalla centralità della cura educativa, si mostrano infatti resistenti all'offerta di occasioni di apprendimento, preoccupati dal rischio di anticipare e affrontare precocemente acquisizioni e conquiste cognitive che i bambini matureranno in un secondo momento, dopo aver pienamente consolidato un'identità psicologica sicura. Chi invece fonda il suo approccio sul principio della competenza socio-cognitiva dei bambini punta soprattutto a cogliere e valorizzare le abilità e le strategie esplorative dei bambini piuttosto delle dimensioni emotivo-relazionali: laboratori, atelier, percorsi e progetti animano le proposte rivolte ai bambini nell'ottica di promuovere un curriculum adeguato all'età dei bambini per sostenere la loro spontanea curiosità e capacità di fare esperienze cognitive e imparare ad imparare.

In entrambe le posizioni, se poste in termini oppositivi in un radicale aut-aut, sono evidentemente presenti rischi e limiti che, a mio parere, stanno conducendo la cultura del nido a una sclerotizzazione pericolosa e non più sostenibile nella sua artificiosità del tutto lontana dalle posizioni più 
recenti della ricerca e soprattutto dalla vita reale dei bambini che invece, se osservati e ascoltati, rivelano quanto nella loro esperienza del mondo naturale, fisico, sociale, l'apprendimento sia intrinseco alla vita emotivo-relazionale e viceversa, quanto sia essenziale fare esperienza di contatti relazionali profondi e significativi con adulti (familiari e professionisti) che sappiano interessarsi e prendersi cura di loro in una prospettiva olistica, attenta alla persona intera, non scissa astrattamente in dimensioni non comunicanti. I bambini hanno bisogno di adulti che sappiano prendersi cura della loro intelligenza (Cagliari, 2017, pag. 16). In altre parole cura, educazione, apprendimenti non sono separabili nella prima infanzia, e nemmeno del resto in tutte le età della vita, né dal punto di vista teorico né tanto meno nelle pratiche educative che educatori e insegnanti pongono in atto sul campo. In una visione olistica dello sviluppo possiamo affermare che la conoscenza umana è intrinsecamente di natura relazionale. I bambini, coinvolti attivamente fin dalla nascita in relazioni improntate alla circolarità e reciprocità con gli adulti che si prendono cura di loro, vivono esperienze di conoscenza del mondo all'interno dei processi emotivo-relazionali cui partecipano e dalle quali imparano. "Capire che qualcuno è interessato a qualcosa del mondo porta i piccoli a fare affidamento sul fatto che c'è qualcosa lì fuori di cui occuparsi “ (Reddy, 2010, pag. 112) ed è quindi partecipando attivamente alla relazione con gli adulti significativi che i bambini costruiscono la loro identità emotivo-cognitivo-sociale aprendosi all'altro e alla realtà. In questa prospettiva non si tratta allora né di sottolineare il valore della cura educativa né tanto meno di evidenziare il rilievo dei processi di sviluppo ma di includere entrambe le dimensioni in una visione relazionale dello sviluppo, improntata alla circolarità e alla reciprocità, e radicata nel contesto reale e vivo della cultura di cui si è parte attiva fin dalla nascita. Rogoff ci ricorda infatti come "in ogni parte del mondo, il bambino, i genitori e gli altri partner strutturano insieme le situazioni cui il bambino partecipa. Il processo di strutturazione riguarda la scelta delle attività cui i bambini possono accedere, assistere e partecipare, e la diretta partecipazione ad attività condivise (conversazioni, racconti, giochi e attività di routine)". (Rogoff, 2004, pagg. 296-297) Se accogliamo questa prospettiva, che sposta l'attenzione sul contesto culturale e sulle pratiche di partecipazione effettivamente messe in atto e condivise da adulti e bambini, l'opposizione tra "cura educativa" e "apprendimenti" si attenua e lascia spazio a nuovi temi di riflessione, più strettamente connessi alle caratteristiche reali e simboliche della vita quotidiana, interrogando gli adulti affinché possano osservare criticamente le scelte e le realizzazioni compiute nel dar forma allimpalcatura che giorno dopo giorno sorregge le esperienze quotidiane. Diventa cioè interessante indagare la struttura del quotidiano come impalcatura di stabilità: "la famiglia, l'asilo nido, la scuola, i gruppi di coetanei così come ogni istituzione o luogo di lavoro, sono caratterizzati da gruppi sociali e sistemi relazionali che esprimono nelle pratiche quotidiane aspetti significativi della loro cultura, rilevanti sul piano psicologico anche per la funzione di supporto alla costruzione della conoscenza di sé, dell'identità, e alla comprensione della realtà circostante" (Emiliani, 2008, pag. 189) E' fondamentale, in questa nuova fase della storia del nido e dei servizi educativi per l'infanzia, che si superino visioni eccessivamente strutturate in adesione a modelli interpretativi polarizzati per elaborare una cultura educativa aperta e critica, capace di lanciare sollecitazioni interessanti ai saperi professionali e alle pratiche proprie della scuola dell'infanzia. Nell'ottica di dar forma a un dialogo proficuo con la cultura maturata dalla scuola dell'infanzia è chiesto, 
credo, alla cultura del nido uno sforzo di superamento critico delle certezze sedimentate, una capacità di problematizzazione e messa in discussione del sapere consolidato per valorizzare, non in termini difensivi ma in chiave innovativa e trasformativa, le acquisizioni educative che puntano sul rispetto e sulla comprensione autentica dei bambini. Molti educatori che operano al nido e negli altri servizi per la prima infanzia sono oggi preoccupati dall'incontro con la scuola, temono di essere catapultati in un orizzonte fatto di didatticismo e nozionismo astratto, e ovviamente di esserne fagocitati. Credo che il punto sia un altro e riguardi non tanto $\mathrm{i}$ timori legati al contatto con il sapere professionale e la tradizione della scuola dell'infanzia quanto piuttosto la qualità, l'autorevolezza e l'attualità della cultura educativa maturata dal nido che, proprio grazie questa nuova apertura definita dalla legge, può essere messa in discussione per costruire, con l'apporto di altri punti di vista professionali, un più ricco sapere educativo sull'infanzia. In questo senso pensare al bambino da zero a sei anni come persona intera, fuori dalla scissione zero-tre/tre-sei, come sollecita la legge, può essere una sfida davvero molto interessante se colta nel suo portato positivo e dialettico, se vista cioè come occasione per educatori e insegnanti di fare ricerca e porsi, per usare il pensiero di Freire, come "ricercatori critici" in un campo, quello educativo, problematico e in divenire, in un incessante che-fare. (Freire, 2002) Nella nuova prospettiva definita dal Decreto n.65, educatori e insegnanti saranno chiamati a progettare insieme, a coordinare il loro lavoro in una logica di continuità nonché a condividere esperienze formative comuni. Sapranno muoversi in quanto ricercatori critici? Sapranno partire dai bambini e dal loro diritto all'educazione in quanto persone intere?

\section{Sguardi e prospettive sui bambini da zero a sei anni}

Si tratta di lavorare alla costruzione di un linguaggio pedagogico comune fondato sulla condivisione di criteri interpretativi in grado di dialogare tra loro per interrogare criticamente le pratiche educative e le esperienze dei bambini. Non significa pensare tutti allo stesso modo, negando le peculiarità e le differenze tra contesti e realtà educative, ma fare riferimento a un'idea di bambino condivisa che permetta a chi opera al nido e alla scuola dellinfanzia di elaborare dei riferimenti riconoscibili e confrontabili per accompagnare in modo sensato e coerente lo sviluppo dei bambini dalla nascita ai sei anni. Ciò che oggi è carente, a cui invita a prestare attenzione e investire la Legge 107, è la messa in atto di una prospettiva di continuità dialettica tra diversi servizi, fatta di imprevisti costruttivi, di svolte utilmente discontinue, di sfide, non certo di piatta e lineare prevedibilità calcolata e diretta dagli adulti, che conduca alla reale esperienza di progettazione e realizzazione di esperienze educative e di apprendimento lungo un continuum significativo e coerente per evitare ai bambini inutili rallentamenti, ripetizioni, accelerazioni o salti forzati, fatiche emotive o cognitive senza senso. A volte questo invece accade anche in piccole circostanze del vivere quotidiano nei servizi quando ad esempio i bambini che hanno già frequentato il nido e maturato sicurezze, abilità, acquisizioni nel campo delle autonomie personali e cognitive devono tornare indietro e ripartire da zero nel contesto della scuola dell'infanzia che li rappresenta come "i piccoli" e, con l'intento di tutelarli, di fatto nega loro ambiti di azione, di esperienza e di movimento indipendente per cui invece questi bambini sarebbero del 
tutto pronti. Gli sguardi e le prospettive educative di educatori e insegnanti sono allora il principale campo di indagine da cui partire e da tematizzare. Per progettare e coordinare azioni e interventi educativi coerenti e condivisi tra educatrici e insegnanti nella logica zero-sei è infatti fondamentale poter disporre di uno sfondo culturale e di un comune repertorio di riferimenti teorico-metodologici cui riferirsi. Significa poter dialogare e confrontare idee, valori, presupposti, criteri educativi non astrattamente ma a partire dai bambini reali nella quotidianità dei contesti educativi e dalle pratiche professionali messe in atto (Infantino, 2014). Significa ad esempio avviare un confronto a partire dall'analisi di una situazione quotidiana come la seguente:

Luca (trenta mesi) insieme ad altri cinque bambini di età compresa tra $\mathrm{i}$ trenta e $\mathrm{i}$ trentasei mesi, con la presenza di due educatrici, è stato osservato per otto minuti in una situazione di "gioco libero" al nido.

I bambini prendono liberamente i giochi dagli scaffali. Luca prende dal mobile la scatola degli incastri "clipo" li travasa in un contenitore e li porta sul tappeto. Prende un po' di clipo della stessa forma, li porta al petto con le mani poi correndo e saltellando li va a mettere su un mobile situato accanto alla porta d'ingresso. Li riprende in mano e corre verso il tappeto, ripete più volte questo tragitto senza posare i pezzi e tenendoli stretti a sé. Si avvicina Paolo e gli chiede "Ne dai uno?" Luca risponde "No!" prende i pezzi di clipo e li porta sul tappeto per poi lasciarli lì. Ora va da Anna che è seduta sul tappeto, salta e sgambetta davanti a lei, Anna gli dice che lui "ha la bua" Luca sgambetta e se ne va via. Prende altri clipo e li butta sul tappeto. Si mette per terra e sgambetta. Paolo ne prende alcuni, Luca si arrabbia e glieli prende. Paolo ne prende uno e lo usa come pistola dicendo "Ban!! Ban!" Luca dice "Ban!!” continuano a dire "Ban!!” imitandosi a vicenda. Le educatrici mettono a disposizione dei bambini stoffe di diverse dimensioni, tubi di cartone, cordicelle di lana, scatole di cartone e metallo, tappi di metallo. Luca prende un rotolo di cartone e lo punta verso la finestra dicendo "Pum! Pum!" poi lo usa come se fosse una mazza da golf sul mucchio delle stoffe sollevandone in aria alcune. Ripete più volte il gesto dicendo in direzione delle educatrici "Guarda!!!" Alcune stoffe rimangono appese al tubo, le va a depositare da un'altra parte. Va da Paolo che ha preso un contenitore dello shampoo e dice "sapone acqua". Paolo versa e Luca fa finta di prendere il contenuto dicendo "ecco fatto". Ricomincia a sollevare stoffe con il tubo, poi va verso una parete su cui sono appese le foto di famiglia di ciascun bambino e rivolgendosi a Paolo: " $\mathrm{E}$ ' mamma tua vero? Ecco fatto, otto nove sette due" indicando intanto le foto. Va a riprendere i cordini e li riporta nel mucchio delle stoffe. Poi torna con il tubo al mobile delle foto di famiglia e col tubo batte sulle foto di famiglia. Quindi va a prendere un contenitore di metallo e lo va a mettere sopra il mobile. Va al tavolo intorno al quale stanno giocando alcuni bambini, prende la bottiglietta di plastica che ha in mano Ilaria insieme a una macchinina. Con la bottiglietta fa finta di riempire la macchina. Prende alcuni pezzi di costruzione dal mobile e cerca di metterli dentro la macchina. Lascia tutto sul tavolo. Gira intorno al tavolo correndo. Trova un coltellino di plastica sopra il mobile e va verso le immagini attaccate al muro vicino alla cucinetta, dice più volte "la mamma" indicando un' immagine femminile, poi indica la figura di un bambino.

Quali significati è possibile cogliere dal comportamento di Luca in questa sequenza osservativa? Cosa vedono gli occhi di educatori e insegnanti? Come tracciare una punteggiatura dei fatti condivisibile per dare forma agli stessi temi e problemi su cui confrontarsi? Questa sequenza, in cui non accade nulla di 
particolarmente eclatante, può aprire innumerevoli spunti di discussione e analisi. Proviamo a evidenziarne alcuni: un primo ambito può riguardare proprio il bambino osservato, per esplorare più in dettaglio i possibili significati che possono essere attribuiti al suo comportamento. Che cosa sta facendo Luca? Come entra in contatto con la realtà, con gli altri bambini e con i materiali che sono a sua disposizione? Sta realizzando un progetto di gioco? Ne diamo una lettura positiva o ci sembra che possano essere intravisti elementi di criticità su cui soffermarsi? Potremmo ragionare sul fatto che questo bambino ha trenta mesi e che in effetti manifesta una serie di abilità, competenze e acquisizioni (nel movimento, nel linguaggio, nelle interazioni sociali) che lo mettono nella condizione di muoversi attivamente nell'ambiente fisico e relazionale. Potremmo anche essere indotti a concludere che del resto, negli otto minuti in cui si è svolta l'osservazione, abbiamo potuto seguire questo bambino in un comportamento di esplorazione, scelta di oggetti, avvio di contatti e scambio con gli altri bambini, che potrebbe denotare una certa sicurezza e intraprendenza. Soprattutto potremmo sottolineare che questo bambino sa muoversi in autonomia, si interessa a una serie di elementi presenti nel contesto e via via si muove nello spazio in una pluralità di sequenze di azione. Ma è questa l'idea di bambino attivo che possiamo condividere? E' questa l'idea di bambino in una logica zero-sei anni che vogliamo costruire? Quali sono gli indicatori che assumiamo per interpretare il comportamento di un bambino come comportamento attivo? Un bambino è attivo e dimostra di essere interessato e coinvolto in un'esperienza anche se solo prende e lascia degli oggetti? Quando iniziamo a discutere su specifiche sequenze di vita dei bambini osservati all'interno dei contesti educativi si creano le premesse per uno scambio profondo su ciò che per noi ha valore nell'educazione dei bambini piccoli, sullidea stessa che abbiamo dei bambini nelle diverse età e nelle esperienze che possono compiere per sperimentare se stessi e il mondo che li circonda. I bambini nel terzo anno di vita sono poi particolarmente interessanti in una prospettiva zero-sei perché si trovano in una "zona di confine" tra zero-tre e tre-sei e portano quindi a formulare questioni e interrogativi chiaramente visualizzabili sia per gli educatori del nido sia per gli insegnanti della scuola dell'infanzia ed è proprio ragionando e discutendo di casi e situazioni concrete, come quella riportata, che diventa possibile percorrere un reale terreno di condivisione pedagogica verso la possibilità di costruire riferimenti condivisi per prendere accordi e progettare azioni educative dotate di un significato riconosciuto. Nel caso specifico del bambino osservato può essere molto interessante ad esempio indagare in modo esplicito come si traccia un confine tra la cosiddetta "libera scelta" del bambino e il vagare senza meta e obiettivo nello spazio, tra l'iniziativa spontanea e il puro prendere e lasciare cose. Quali indicatori rivelano attenzione e concentrazione in un bambino di trenta mesi? Quali indicatori rivelano invece il suo girare a vuoto? Cosa pensiamo dei bambini nel loro terzo anno di età? Quali proposte ha senso che siano pensate e allestite specificatamente per loro? Quali abilità e competenze meritano di essere colte e sostenute in questa fase della vita dei bambini? $\mathrm{E}$ in particolare nel caso di questo bambino, che-fare? $\mathrm{E}$ a questo punto si aprono altri scenari di riflessione che riguardano più direttamente il ruolo educativo degli adulti. Quando intervenire (e come?) e quando aspettare e dare tempo ai bambini? In questo caso specifico le educatrici sono rimaste in ombra. Come mai? E' questo il modo condivisibile di intendere e praticare il ruolo dell'educatore e dell'insegnante? Se, in una prospettiva zero-sei, pensiamo ai bambini non solo nel loro 
presente ma anche nell'evoluzione delle loro esperienze allora è importante condividere quale debba essere il ruolo e l'intervento educativo degli adulti in relazione ai comportamenti, ai bisogni, ai desideri che possiamo cogliere o ipotizzare da parte dei bambini. Scambiare idee, condividere finalità educative, prendere accordi e condividere pratiche intenzionali: educatrici e insegnanti sono pronte a una condivisione di questo tipo? Lavorare in una prospettiva zero-sei è una sfida impegnativa, per la quale sarà necessario un investimento culturale importante che non potrà essere interamente compiuto dai soli educatori e insegnanti ma richiederà l'attivazione di strategie di coordinamento dei servizi molto mirate nonché di finalizzati processi di formazione in servizio. Anche su questi aspetti si è espresso il Decreto n.65 nello specifico assegnando alle Regioni e agli Enti Locali (nell'articolo 6 e nell'articolo 7) il compito di promuovere e attivare i "coordinamenti pedagogici territoriali" per il supporto professionale al personale del Sistema integrato di educazione e di istruzione.

\section{Coordinare e formare la professionalità di educatori e insegnanti}

La ricerca in questi anni ha confermato, (Bove, Braga e Mantovani, 2016) quanto il coordinamento e la formazione in servizio giochino un ruolo cruciale sulla qualità della professionalità di educatori e insegnanti nonché del servizio nel suo complesso. In una svolta così rilevante per il sistema dei servizi rivolti all'infanzia, il coordinamento e la formazione rappresentano due nodi nevralgici per la riuscita di un processo di così ampia portata e su questi due versanti dovrebbe quindi essere concentrato un investimento mirato, consistente, sistematico. Non si può infatti sostenere una nuova fase culturale e una nuova organizzazione del sistema di educazione e istruzione senza un solido coordinamento dei servizi, dei gruppi di lavoro e delle interazioni tra le diverse realtà educative. E' tuttavia proprio nella definizione del coordinamento del sistema educativo che l'assetto legislativo appare meno chiaro e esplicito rinviando la questione (come del resto è corretto che sia) alle Regioni e agli Enti locali ai quali compete non solo una più specifica definizione ma anche l'articolazione sul territorio. Ed è a questo livello che si rischia, a mio parere, di far vacillare la spinta innovativa e trasformativa contenuta nel dispositivo legislativo lasciando che le volontà politiche, variabili e fortemente diversificate come già accennato da regione a regione, possano avere una chiara preponderanza nella realizzazione effettiva o solo su carta del sistema integrato zero-sei. Il rischio in altre parole è che, senza una chiara definizione delle articolazioni delle funzioni di coordinamento, il sistema integrato zero-sei potrà avere piena realizzazione in quelle regioni virtuose che hanno già una buona offerta di servizi e un articolato sistema, con una solida

governance pubblica; nelle altre regioni, dove si registra il mancato decollo dei servizi per l'infanzia, il sistema integrato, senza un sistematico impianto di coordinamento, potrebbe invece restare un obiettivo del tutto irraggiungibile. Le ragioni per cui il coordinamento gioca un peso così decisivo sull'attivazione del sistema integrato dei servizi si articolano, a mio parere, su due ordini di considerazioni a cui in estrema sintesi richiamo l'attenzione. La prima riguarda le dimensioni micro-contestuali dei singoli servizi: le funzioni di coordinamento a questo livello si traducono in forme di presenza, diretto contatto e intervento all'interno della quotidianità dei servizi e sono garanzia della traduzione coerente del progetto educativo in azioni e pratiche di qualità. La 
seconda, a livello macro-contestuale: le funzioni di coordinamento promuovono collaborazioni e connessioni sinergiche tra le realtà educative e scolastiche del territorio tanto più essenziali quanto più si considera la natura complessa del sistema zero-sei composto da servizi gestiti direttamente dal pubblico, dal privato, dal privato sociale e dallo stato. Senza una regia pedagogica autorevole, definita, continuativa e sistematica in grado di coordinare questi due livelli il sistema zero-sei sarebbe privato delle connessioni interattive imprescindibili per essere effettivamente realtà sistemica. In questo senso il dispositivo legislativo più che permettere un ventaglio di possibili declinazioni, che possano essere percorribili, compatibili e sostenibili nelle diverse realtà, rischia di non assegnare il necessario valore alle funzioni di coordinamento dal momento che non è chiaro se avviarsi verso un unico coordinamento (con elevati livelli di complessità nel caso si tratti ad esempio di nido comunale e scuola infanzia statale), oppure verso più figure di coordinatori sui diversi servizi che condividono le funzioni di coordinamento zero-sei, valorizzando e integrando le competenze delle risorse professionali già presenti sul territorio. L'elemento che il dettato legislativo definisce in modo univoco è il carattere pedagogico del coordinamento stabilendo in tal modo la rilevanza di un profilo e di un quadro di competenze professionali molto chiaro per svolgere funzioni e interventi nei servizi in un campo di intervento esplicitamente educativo e non solo, come spesso accade, esclusivamente o prevalentemente amministrativo-gestionale. Definire in termini pedagogici le funzioni assegnate al coordinamento richiama positivamente a una serie di ambiti di intervento di grande rilievo che riguardano: l'istituzione e la definizione di un gruppo di lavoro, di cui il coordinatore è responsabile e garante; l'individuazione e assegnazione di obiettivi verso cui il gruppo di lavoro orienta le proprie attività; l'adozione di un metodo di lavoro che consenta la progettazione, la realizzazione, la documentazione e l'analisi di intervento educativi condivisi; la messa in atto di processi di monitoraggio e valutazione del lavoro svolto. Solo un coordinamento con specifiche competenze pedagogiche può adeguatamente svolgere queste funzioni essenziali per alimentare la vita di gruppi di lavoro attivi e capaci di condividere una cultura educativa innovativa. L'altro polo rilevante quando si riflette sulle funzioni di coordinamento concerne la formazione in servizio. Questo potrebbe essere colto come ulteriore passaggio critico del Decreto 65 della Legge 107 se si considera che educatori e insegnanti avranno svolto percorsi di formazione di base universitaria distinti e specifici: per gli educatori nei servizi zero-tre è previsto un percorso di studi di durata triennale in Scienze dell'Educazione a indirizzo specifico per educatore di nido; per gli insegnanti della scuola dell'infanzia invece un percorso quinquennale in Scienze della Formazione Primaria, prevedendo la possibilità di accedere, eventualmente, anche al lavoro nei servizi educativi conseguendo altri sessanta crediti formativi in un percorso universitario specificamente definito. Non è questa la sede per un'analisi approfondita dei diversi percorsi di formazione universitaria, ma da queste brevi indicazioni dovrebbe comunque poter emergere la distanza che separa i due percorsi universitari. Alla formazione in servizio si chiede dunque un compito decisivo rispetto alla creazione di una cultura zero-sei. Spetta infatti alla formazione in servizio il ruolo di avvicinare, far dialogare e convergere in una cultura pedagogica comune educatori e insegnanti che la formazione universitaria ha tenuto separati in percorsi formativi distinti e che, impegnati nel lavoro sul campo, operano in servizi diversi. Nel complesso non si può che sottolineare quanto il dispositivo formativo mostri non poche incongruenze e problematicità. Potrà la formazione in servizio attivare contesti e 
processi in grado di sostenere efficacemente educatori e insegnanti verso una nuova cultura educativa zero-sei? Riuscirà ad assolvere a funzioni trasformative e generative di nuovi pensieri e pratiche educative di qualità? La posta in gioco e il livello della sfida sono davvero molto alte.

\section{Referências}

BOVE, Chiara; BRAGA, Piera; MANTOVANI, Susanna. Videoricerca e formazione. Un approccio interculturale. In BOBBIO, Andrea, TRAVERSO, Andrea (a cura di). Contributi per una pedagogia dell'infanzia. Teorie, modelli, ricerche. Bologna: Edizioni ETS, 2016. Pp. 147-173

CAGLIARI, Paola. Costruire un curricolo 0/6: un'occasione per definire significati e concetti trasversali. In LICHENE, Claudia (a cura di). Conoscere lo 0-6. Bergamo: Zeroseiup edizioni, 2017. pp. 13-23

EMILIANI, Francesca. La realtà delle piccole cose. Psicologia del quotidiano. Bologna: Il Mulino, 2008. (tem versão em português)

FREIRE, Paulo. La pedagogia degli oppressi. Torino: EGA, 2002 (tem versão em português)

INFANTINO, Agnese (a cura di) Pratiche educative nei servizi per l'infanzia. Milano: Franco Angeli, 2014

REDDY, Vasudevi. Cosa passa per la testa di un bambino. Emozioni e scoperta della mente. Milano: Raffaello Cortina editore, 2010

ROGOFF, Barbara. La natura culturale dello sviluppo. Milano: Raffaello Cortina Editore, 2004. (tem versão em português)

Legge 15 luglio 2015 n. 107 "Riforma del sistema nazionale di istruzione e formazione e delega per il riordino delle disposizioni legislative vigenti”, Gazzetta Ufficiale, serie generale, n. 162

Indicazioni Nazionali per il curricolo della scuola dell'infanzia e del primo ciclo d'istruzione, Settembre 2012

Decreto Legislativo 13 aprile 2017 n. 65, Gazzetta Ufficiale n. 112 del 16.05.2017, Supplemento Ordinario n.23 